\title{
Usefulness of a crista catheter for 3-dimensional electroanatomical mapping of complex right atrial tachyarrhythmias
}

\author{
Jae-Sun Uhm ${ }^{1}$ • Nam Kyun Kim ${ }^{2}$ - Hancheol Lee ${ }^{1}$ - Tae-Hoon Kim ${ }^{1}$ • Boyoung Joung ${ }^{1}$. \\ Hui-Nam Pak ${ }^{1}$ - Moon-Hyoung Lee ${ }^{1}$
}

Received: 26 May 2015 / Accepted: 30 July 2015 /Published online: 11 August 2015

(C) The Author(s) 2015. This article is published with open access at Springerlink.com

\begin{abstract}
Purpose Right atrial (RA) tachyarrhythmias are not rare in patients with congenital heart disease and a history of cardiac surgery. This study investigated the usefulness of a crista catheter for 3-dimensional electroanatomical mapping of RA tachyarrhythmias.

Methods We consecutively included 35 patients (age, $43.2 \pm$ 15.6 years; 15 men) who underwent an electrophysiological study with 3-dimensional electroanatomical mapping for RA tachycardia or flutter. In 13 patients with atrial flutter, we recorded and compared the electrical sequence in the anterior and posterior portions of the RA lateral wall. We used a crista catheter as a mapping catheter for 3-dimensional mapping in 12 patients (crista group), a lasso catheter in 12 patients (lasso group), and an ablation catheter in 11 patients (ablation group). We compared the 3-dimensional mapping points, time, and speed (mapping points per minute) among the groups.

Results Atrial flutter was confirmed as cavotricuspid isthmusdependent in all patients whose two atrial electrical sequences were the same direction and as atypical (including scar-related and dual-loop) in all patients whose sequences were in the opposite direction. Mapping speed in the crista group was significantly faster than in the lasso and ablation groups: median (interquartile range) 44.0 (35.5-69.4) points/min, 23.7
\end{abstract}

Moon-Hyoung Lee

mhlee@yuhs.ac

1 Department of Cardiology, Severance Cardiovascular Hospital, Yonsei University College of Medicine, 50-1 Yonsei-ro Seodaemun-gu, Seoul, South Korea 120-752

2 Department of Pediatric Cardiology, Severance Cardiovascular Hospital, Yonsei University College of Medicine, 50-1 Yonsei-ro Seodaemun-gu, Seoul, South Korea
(17.8-29.8) points/min, and $8.2(4.8-11.0)$ points $/ \mathrm{min}$, respectively $(p=0.001)$.

Conclusions A crista catheter is useful for high-density 3-dimensional electroanatomical mapping of complex RA tachyarrhythmias. Comparison of the electrical sequences in the anterior and posterior portions of the RA lateral wall is helpful for differentiating between cavotricuspid isthmus-dependent and atypical atrial flutter.

Keywords Atrial flutter · Atrial tachycardia $\cdot$ Crista catheter . Right atrium $\cdot$ Three-dimensional mapping

\section{Introduction}

The incidence of right atrial (RA) tachycardia is higher than that of left atrial tachycardia [1,2]. RA flutter in patients with congenital heart disease is not rare [3]. The mechanisms of atrial tachycardias include macro- and micro-reentry, triggered activity, and enhanced automaticity [4, 5]. It is often difficult to control atrial tachycardia and atypical atrial flutter with medication [6]. Radiofrequency catheter ablation (RFCA) is a good treatment option for sustained atrial tachycardia and flutter. The origin and the mechanism of atrial tachycardias can be determined using surface electrocardiography (ECG) and conventional mapping methods. In addition to conventional mapping, 3-dimensional (3D) electroanatomical mapping systems are very helpful in patients with atypical atrial flutter or complex heart anatomy, including those with congenital heart disease or a history of cardiac surgery [7]. Although an ablation catheter or a lasso catheter is often used for 3D mapping, it is not easy to map the RA with either of these types of catheter. A crista catheter is designed for mapping of the crista terminalis area and has multiple electrodes with narrow spaces between them. The purpose of this study was to 
assess the usefulness of a crista catheter for $3 \mathrm{D}$ electroanatomical mapping of the RA.

\section{Methods}

We consecutively included 35 patients (age, $43.2 \pm 15.6$ years; 15 men) who underwent an electrophysiological study and RFCA with use of a 3D electroanatomical mapping system (CARTO, Biosense Webster Inc., Diamond Bar, CA, USA, or EnSite NavX, St. Jude Medical Inc., Minnetonka, MN, USA) for symptomatic sustained RA tachycardia and flutter between January 2011 and August 2014. Each patient provided written informed consent. All antiarrhythmic drugs were discontinued for at least five times of half-lives prior to the electrophysiological study. Oral anticoagulants were administered according to the physicians' discretion. In patients with complex arrhythmias or unusual heart anatomy, contrastenhanced cardiac computed tomography was performed using a dual-source scanner (Somatom Definition Flash, Siemens Healthcare, Forchheim, Germany) for 3D electroanatomical mapping prior to the electrophysiological study.

The electrophysiological study was performed with the patient in a fasting state, under local anesthesia with mild-tomoderate sedation. Figure 1 shows three examples of the usual placement of the electrophysiological catheters during the electrophysiological study. Twelve-lead surface ECG and intracardiac electrogram recordings were displayed and stored on a computer-based digital amplifier system (Prucka Systems, General Electric Healthcare Inc., Milwaukee, WI, USA, or WorkMate, St. Jude Medical Inc.). During sustained atrial tachycardia or flutter, we mapped the tachycardia using conventional methods and a 3D electroanatomical system. In cases of atrial flutter, we placed a crista catheter or a deflectable duodecapolar catheter in the RA and recorded the intracardiac electrogram in the anterior and posterior portions of the RA lateral wall, before 3D mapping was performed. When the two sequences of the atrial electrogram were in the same direction, we considered cavotricuspid isthmus (CTI)-dependent atrial flutter to be highly probable, whereas when the two sequences were in opposite directions, we judged there to be a high probability of atypical atrial flutter, including scar-related or dual-loop atrial flutter. Conventional methods included activation mapping and entrainment pacing. As mapping catheter for 3D electroanatomical mapping, we used a 20-pole deflectable crista catheter in 12 patients (crista group; Fig. 1a), a 20-pole deflectable lasso catheter (Biosense Webster Inc.) in 12 patients (lasso group; Fig. 1b), and a 4-pole deflectable ablation catheter (ThermoCool Catheter, Biosense Webster Inc.) in 11 patients (ablation group; Fig. 1c) as a mapping catheter. The crista catheter is 7 French in size and has 201 $\mathrm{mm}$ electrodes with 1-3-1 mm inter-electrode spacing and a D-shaped deflection which is suitable to be positioned along
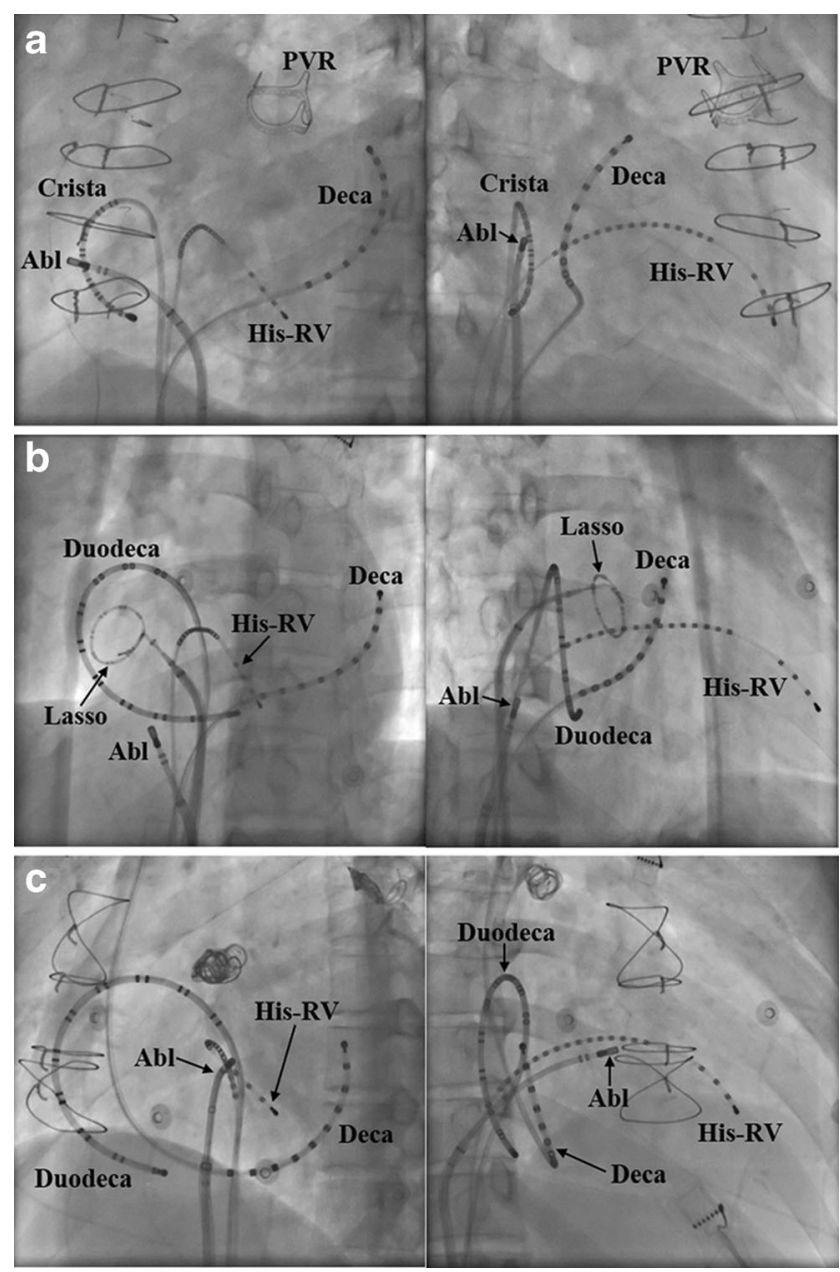

Fig. 1 a Usual catheter position in the crista group. Left and right anterior oblique fluoroscopic views show a crista catheter (Crista) in the right atrium, a decapolar catheter (Deca) in the coronary sinus, a His-RV type catheter $(H i s-R V)$ in the right ventricle, and an ablation catheter $(A b l)$ in the right atrium. b Usual catheter position in the lasso group. Left and right anterior oblique fluoroscopic views show a lasso catheter (Lasso), a duodecapolar catheter (Duodeca), and an ablation catheter in the right atrium, a decapolar catheter in the coronary sinus, and a His-RV-type catheter in the right ventricle. c Usual catheter position in the ablation group. Left and right anterior oblique fluoroscopic views show a duodecapolar catheter and an ablation catheter in the right atrium, a decapolar catheter in the coronary sinus, and a His-RV-type catheter in the right ventricle. $P V R$ pulmonic valve replacement

the crista terminalis. An SL-1 or SR-0 sheath (St Jude Medical Inc.) was used to introduce the mapping catheter, according to the operator's preference. During the clinical tachycardia, an activation map and a voltage map were created with the mapping catheter. The 3D mapping time and points were measured and recorded. After activation and voltage mapping, in cases of macroreentry tachycardia, entrainment pacing was performed at the potential critical isthmuses. After the origin and mechanisms of tachycardia had been identified using conventional methods and the 3D electroanatomical mapping system, radiofrequency energy was delivered at the points of earliest electric activity in cases of focal atrial tachycardia, or 
the slow-conducted critical isthmus in cases of macroreentrant tachycardia. The maximum power output of the radiofrequency energy was $40 \mathrm{~W}$ using a 3.5 -mm irrigated-tip ablation catheter (ThermoCool Catheter, Biosense Webster Inc.).

The endpoint of the electrophysiological study was termination of the tachycardia during radiofrequency energy delivery and non-inducibility of the tachycardia during atrial burst pacing and isoproterenol infusion of $2-4 \mu \mathrm{g} / \mathrm{min}$. Fluoroscopy time and procedure time were recorded. Acute success was defined as achievement of the endpoint during the electrophysiological study. Major complications were defined as hemopericardium/cardiac tamponade, vascular access site bleeding that required transfusion, advanced atrioventricular block, symptomatic sinus node dysfunction, and phrenic nerve palsy. The patients were followed up and monitored in the outpatient clinic. If there was no evidence of tachycardia, all antiarrhythmic drugs were discontinued. Recurrence was defined as ECG-documented sustained tachycardia with the same $\mathrm{P}$ wave or $\mathrm{F}$ wave morphology.

\subsection{Statistical analysis}

We compared mapping points, time, and speed (mapping points per minute) during the $3 \mathrm{D}$ electroanatomical mapping among the three groups. We also compared the fluoroscopy time and procedure time of the electrophysiological study among the three groups. We used nonparametric methods because these data were distributed non-normally. The results are expressed as median (interquartile range) for continuous variables and count (percentage) for categorical variables. We compared the parameters among three groups using the Kruskal-Wallis test for continuous data and Fisher's exact test for categorical data. We compared the parameters between two groups using the Mann-Whitney test for continuous data. A $p$ value $<0.05$ was considered significant. The data were analyzed using the Statistical Package for the Social Sciences, version 20.0 (IBM Inc., Armonk, NY, USA).

\section{Results}

Table 1 shows the baseline characteristics of the patients according to group. Among the total of 35 patients, 16 had undergone cardiac surgery for congenital heart disease and 4 had undergone valve replacement surgery for acquired valvular heart disease. In all patients who had undergone cardiac surgery, right atriotomy had been performed for approach or cannulation. In the patients who had undergone cardiac

Table 1 Baseline characteristics in the three groups

\begin{tabular}{llll}
\hline & Crista group $(n=12)$ & Lasso group $(n=12)$ & Ablation group $(n=11)$ \\
\hline Age (years) & $48.5(33.3-62.8)$ & $38.5(22.0-50.8)$ & $45.0(31.0-52.0)$ \\
Male sex, $n(\%)$ & $9(75.0)$ & $3(25.0)$ & $3(27.3)$ \\
Cardiac surgery, $n(\%)$ & $7(58.3)$ & $6(50.0)$ & $7(63.6)$ \\
Valve replacement, $n(\%)^{\mathrm{a}}$ & $1(8.3)$ & $3(25.0)$ & $0(0.0)$ \\
Congenital heart disease, $n(\%)$ & $6(50.0)$ & $3(25.0)$ & $7(63.6)$ \\
ASD, $n(\%)$ & $1(8.3)$ & $2(16.7)$ & $1(9.1)$ \\
VSD, $n(\%)$ & $0(0.0)$ & $0(0.0)$ & $1(9.1)$ \\
TOF, $n(\%)$ & $2(16.7)$ & $0(0.0)$ & $2(18.2)$ \\
ccTGA, $n(\%)$ & $1(8.3)$ & $0(0.0)$ & $1(9.1)$ \\
cTGA, $n(\%)$ & $0(0.0)$ & $1(8.3)$ & $0(0.0)$ \\
PA, $n(\%)$ & $1(8.3)$ & $0(0.0)$ & $0(0.0)$ \\
DORV, $n(\%)$ & $1(8.3)$ & $0(0.0)$ & $0(0.0)$ \\
Ebstein anomaly, $n(\%)$ & $0(0.0)$ & $0(0.0)$ & $1(9.1)$ \\
Single ventricle, $n(\%)$ & $0(0.0)$ & $0(0.0)$ & $1(9.1)$ \\
Tachycardia in EPS & & & $6(54.5)$ \\
Focal AT, n $(\%)$ & $6(50.0)$ & $3(25.0)$ & $1(9.1)$ \\
CTI-dependent AFL, $n(\%)$ & $2(16.7)$ & $3(25.0)$ & $3(27.3)$ \\
Scar-related AFL, $n(\%)$ & $2(16.7)$ & $3(25.0)$ & $1(9.1)$ \\
Dual-loop AFL, $n(\%)$ & $2(16.7)$ & $3(25.0)$ & \\
\hline
\end{tabular}

Continuous data are expressed as median (interquartile range)

$A F$ atrial fibrillation, $A F L$ atrial flutter, $A S D$ atrial septal defect, $A T$ atrial tachycardia, $c c T G A$ congenitally corrected transposition of the great arteries, $C T G A$ complete transposition of the great arteries, $C T I$ cavotricuspid isthmus, DORV double-outlet right ventricle, EPS electrophysiological study, $P A$ pulmonary atresia, TOF tetralogy of Fallot, VSD ventricular septal defect

${ }^{a}$ Valve replacement surgery for acquired valvular heart disease 


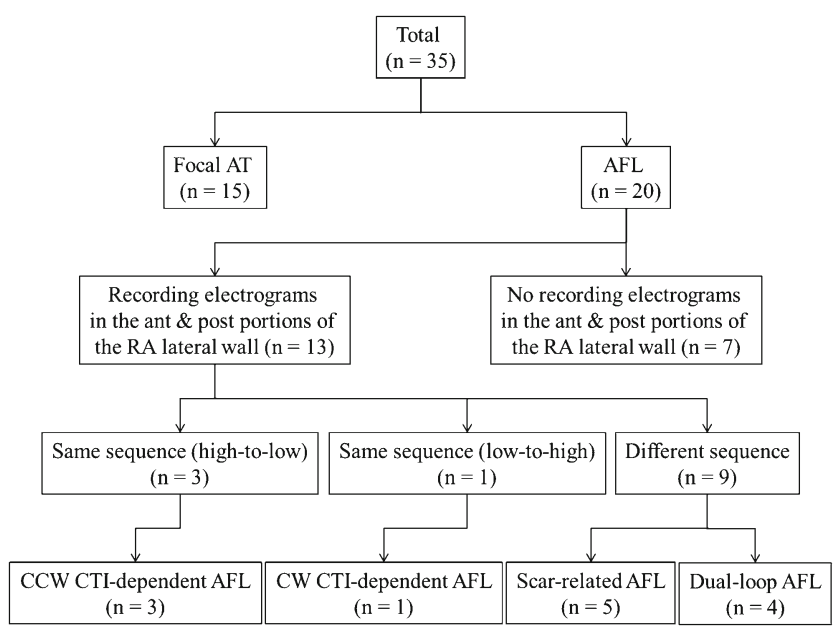

Fig. 2 Flow diagram. $A F L$ atrial flutter, $A T$ atrial tachycardia, $C C W$ counterclockwise, $C T I$ cavotricuspid isthmus, $C W$ clockwise, $R A$ right atrium surgery, the duration between cardiac surgery and the onset of tachycardia was 16.2 (8.1-27.1)years. Electrophysiological studies revealed focal atrial tachycardia, CTI-dependent, scar-related, and dual-loop atrial flutter in 15, 6, 8, and 6 patients, respectively. The six patients with dual-loop atrial flutter included CTI-dependent and scar-related reentrant tachycardia. In 13 of the 14 patients with scar-related or dual-loop atrial flutter, right atriotomy scar was related to the reentry circuit, while in 1 patient, idiopathic scar in the RA was related to the reentry circuit.

In 13 of the 20 patients with atrial flutter on the surface ECG, we recorded and compared the sequence of the atrial electrograms in the anterior and posterior portions of the RA lateral wall (Fig. 2). In four patients, the two sequences of the atrial electrogram had the same as high-to-low or low-to-high direction. In three of these four patients, the two sequences were in high-to-low; the tachycardias were confirmed by $3 \mathrm{D}$
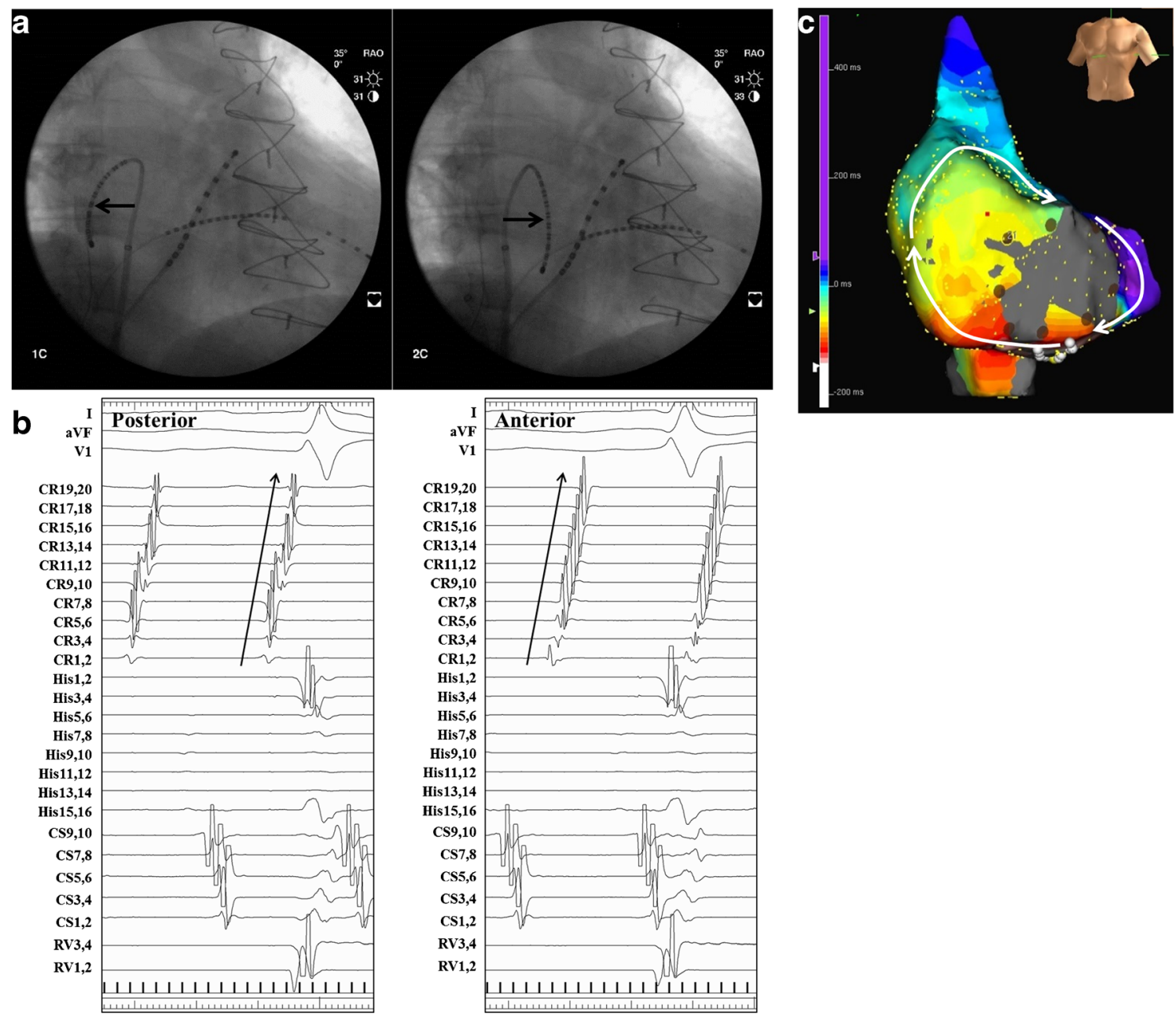

Fig. 3 Clockwise cavotricuspid isthmus-dependent atrial flutter in a 40year-old female patient who underwent total correction of tetralogy of Fallot. a Right anterior oblique fluoroscopic views show the crista catheter (arrow) located in the posterior and anterior portions of the RA lateral wall, respectively. b Both electrograms from the posterior and anterior

portions of the RA lateral wall show low-to-high sequence. c Threedimensional activation map in left anterior oblique view. White arrows indicate electric activation sequence. White and brown dots indicate ablation sites and the tricuspid annulus, respectively 
electroanatomical mapping as counterclockwise CTIdependent atrial flutter. In one of these four patients, the two sequences were in low-to-high; the tachycardias were confirmed as clockwise CTI-dependent atrial flutter (Fig. 3). In these four patients, the atrial flutter was terminated by the CTI block. In nine patients, the two sequences of the atrial electrogram were in opposite directions (high-to-low and low-tohigh; Fig. 4). In five of these nine patients, the tachycardias were confirmed by $3 \mathrm{D}$ electroanatomical mapping as scar-related atrial flutter with a reentry circuit around the right atriotomy scar; the atrial flutter was terminated by linear ablation between the inferior margin of the scar and the inferior vena cava. In the other four patients, the tachycardias were confirmed by $3 \mathrm{D}$ electroanatomical mapping as dual-loop atrial flutter, including scar-related and CTI-dependent atrial flutter, and the atrial flutter was terminated by the CTI block and the linear ablation between the inferior margin of the scar and the inferior vena cava. When we carefully recorded the electrogram around the RA lateral wall with a crista catheter or a duodecapolar catheter during tachycardia in patients with scar-related atrial flutter, we were able to record the turnaround point of electric activation at the inferior margin of the right atriotomy scar (Fig. 5). In this case, atrial flutter was terminated by linear ablation between the turnaround point and the inferior vena cava.

Table 2 shows parameters related to the $3 \mathrm{D}$ electroanatomical mapping and outcomes according to group. The crista group had significantly more mapping points than did the lasso group or the ablation group $(p<0.001)$. The mapping time in the crista group and the lasso group was significantly shorter than in the ablation group $(p<0.001)$. The mapping speed in the crista group was significantly faster than in the lasso group or the ablation group [44.0 (35.5-69.4) points/ min, $23.7(17.8-29.8)$ points/min, and $8.2(4.8-11.0)$ points/
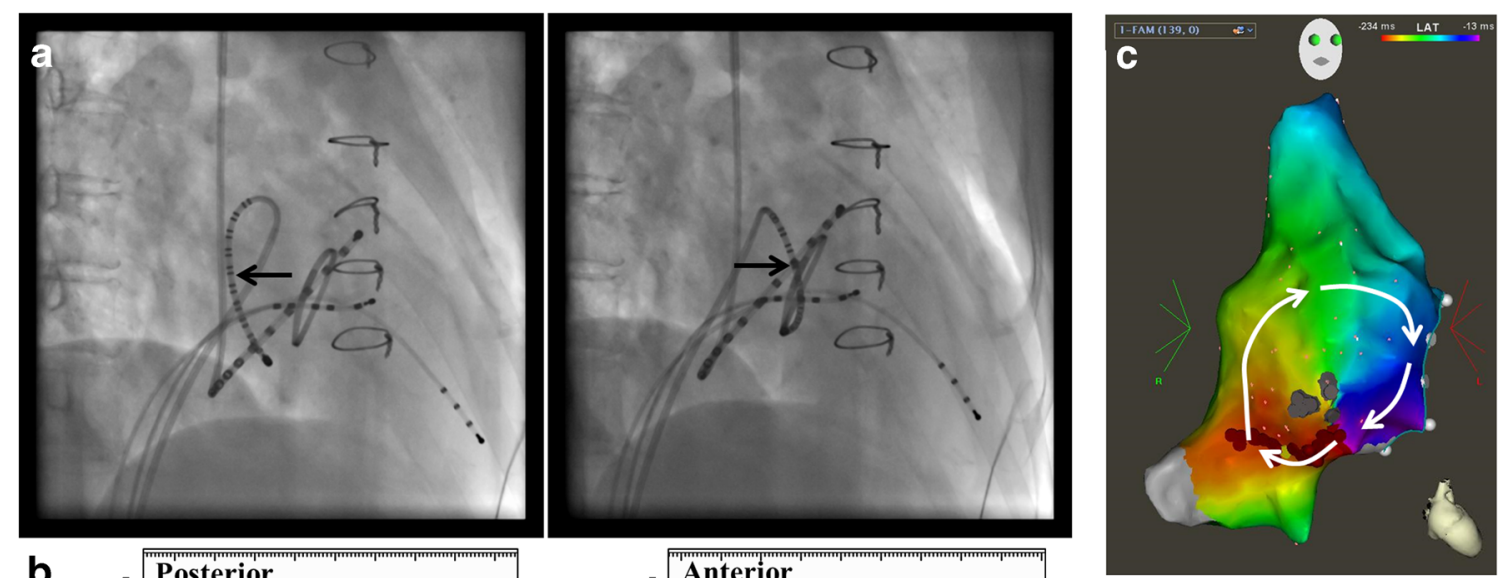

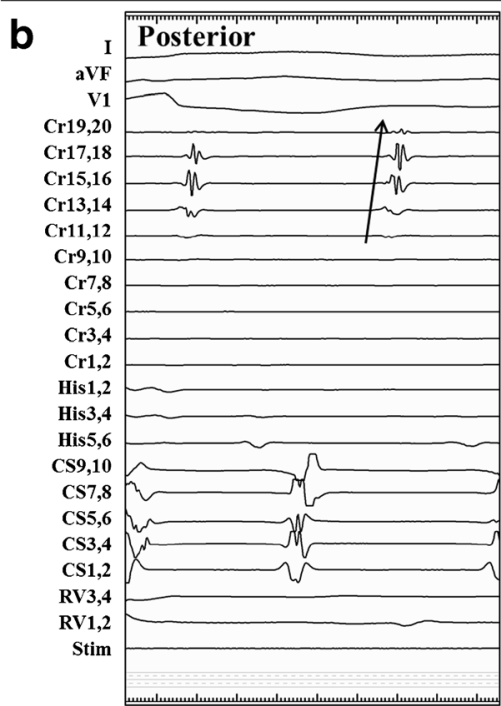

Fig. 4 Scar-related atrial flutter in a 31-year-old female patient who underwent atrial septal defect repair. a Right anterior oblique fluoroscopic views show the crista catheter (arrow) located in the posterior and anterior portions of the RA lateral wall, respectively. $\mathbf{b}$ The electrogram from the posterior portion of the RA lateral wall shows low-to-high sequence,

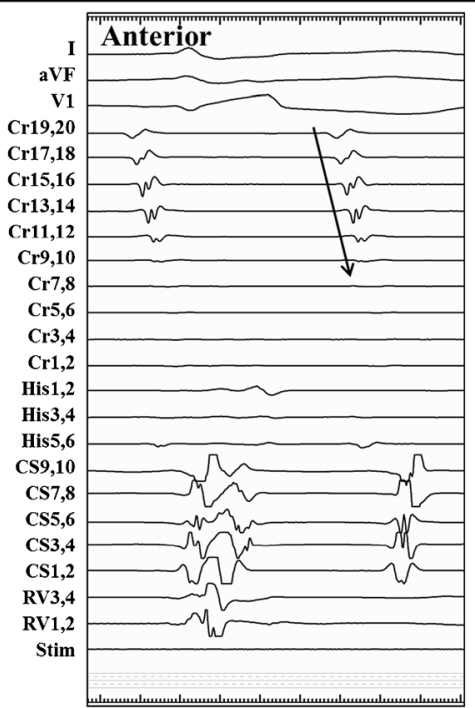

whereas the electrogram from the anterior portion shows high-to-low sequence. c Three-dimensional activation map in right anterior oblique view. White arrows indicate electric activation sequence. White, gray, and red dots indicate the tricuspid annulus, scar tissue, and ablation sites, respectively 
min, respectively; $p=0.001$; Fig. 6]. Fluoroscopic time in the crista group and the lasso group was significantly shorter than in the ablation group ( $p=0.009$ ). Procedure time in the crista group and the lasso group was shorter than in the ablation group ( $p=$ $0.031)$. There were no major complications during the procedure. There were no significant differences in acute success rate among the groups $(p=0.636)$. After RFCA, the patients were followed up for $15.2 \pm 14.5$ months. There were no significant differences in recurrence rate among the groups $(p=0.523)$.

\section{Discussion}

The present study showed that 3D electroanatomical mapping with a crista catheter was faster and denser than with a lasso catheter or an ablation catheter in patients with RA tachyarrhythmias. In addition, the comparison of two electrical sequences from the anterior and posterior portions of the RA lateral wall was helpful for differentiating between the CTIdependent and atypical atrial flutter.


Fig. 5 Scar-related atrial flutter in a 40-year-old female patient with functional single ventricle. a Electrograms in the posterior portion of the RA lateral wall, the right atriotomy scar, and the anterior portion of the RA lateral wall show high-to-low, the turnaround point (asterisk), and low-to-high, respectively. b Left and right anterior oblique fluoroscopic views. A duodecapolar catheter (Duodeca) and an ablation catheter $(A b l)$ were placed at the right atrium. A decapolar catheter (Deca) and a quadripolar catheter (Quadri) were placed in the coronary sinus and at the ventricle, respectively. Solid-line arrows indicate the reentry circuit and direction of the electric activation. A broken-line arrow indicates the passive conduction from the reentry circuit. An asterisk indicates the turnaround point of the electric activation 
Table 2 Parameters related to 3-dimensional electroanatomical mapping and outcomes according to group

\begin{tabular}{|c|c|c|c|c|c|}
\hline & Crista group $(n=12)$ & Lasso group $(n=12)$ & Ablation group $(n=11)$ & $p$ value $^{\mathrm{a}}$ & $p$ value $^{\mathrm{b}}$ \\
\hline Mapping points & $475.0(366.3-569.0)$ & $338.5(166.3-404.8)$ & $162.0(109.1-185.0)$ & $<0.001$ & 0.021 \\
\hline Mapping time (min) & $9.0(7.5-12.3)$ & $12.5(9.5-18.5)$ & $24.0(15.0-27.0)$ & $<0.001$ & 0.069 \\
\hline Fluoroscopy time (min) & $36.0(25.5-39.5)$ & $33.0(19.0-36.5)$ & $53.0(37.0-67.0)$ & 0.009 & 0.050 \\
\hline Procedure time (min) & $156.0(120.0-196.5)$ & $147.5(107.8-165.8)$ & $240.0(167.0-304.0)$ & 0.031 & 0.099 \\
\hline Major complication, $n(\%)$ & $0(0.0)$ & $0(0.0)$ & $0(0.0)$ & $>0.999$ & - \\
\hline Acute success, $n(\%)$ & $12(100.0)$ & $12(100.0)$ & $10(90.9)$ & 0.636 & - \\
\hline Recurrence, $n(\%)$ & $1(8.3)$ & $1(8.3)$ & $1(9.1)$ & 0.523 & - \\
\hline
\end{tabular}

Continuous data are expressed as median (interquartile range)

${ }^{\text {a }}$ Comparison among the three groups

${ }^{\mathrm{b}}$ Comparison between the crista group and the lasso group

In patients who have congenital heart disease or who have undergone cardiac surgery via a right atriotomy, reentrant tachycardia originating from the RA is common $[3,8]$. It is challenging and time-consuming to perform an electrophysiological study in patients with congenital heart disease or a history of cardiac surgery [8]. It is not rare for tachycardias with multiple origins or multiple reentrant circuits to be induced after RFCA of the first tachycardia in these patients [9-11]. During 3D electroanatomical mapping, we can usually use an ablation catheter or a lasso catheter as a mapping catheter. However, 3D mapping of the RA with these types of catheter is difficult and time-consuming. This is because the lasso catheter was originally designed for mapping of tubular structures, including the pulmonary veins, while an ablation catheter has only a small number of electrodes. A 20-pole spiral mapping catheter (A Focus, St. Jude Medical Inc.) and

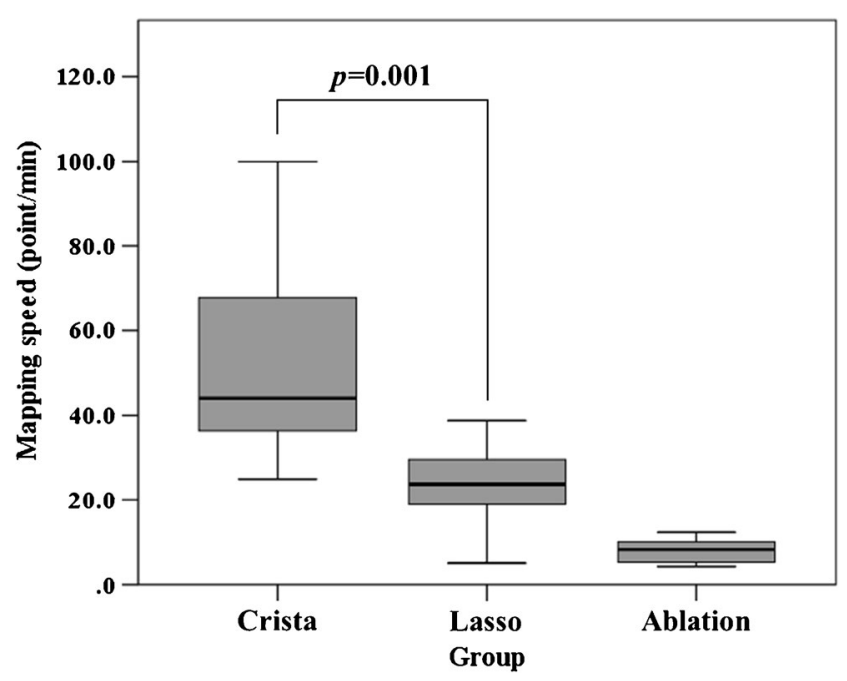

Fig. 6 Three-dimensional electroanatomical mapping speed according to group. Mapping speed was significantly faster in the crista group than in the lasso group and the ablation group. Mapping speed (point $/ \mathrm{min})=$ mapping point/mapping time a 20-pole deflectable pentaray catheter (Biosense Webster Inc.) can be used for 3D mapping [12-14]. However, the spiral mapping catheter, like a lasso catheter, was originally designed for mapping the pulmonary veins. A newly emerging pentaray catheter is a pentagon-shaped mapping catheter with 1-mm electrodes and 2-mm inter-electrode spacing. We can obtain 3D mapping of four chambers with high resolution [15]. A pentaray catheter remains to be validated for RA mapping. Because the crista catheter was designed for recording electric signals from the crista terminalis area, the catheter shape is round and is suitable for mapping the lateral wall of the RA. If a crista catheter is rotated in the RA, the electrical signals can be rapidly recorded from the posterior and septal walls. Because the crista catheter has 20 electrodes with only $1 \mathrm{~mm}$ of space between them, high-density activation mapping can be performed within a short time. In particular, 3D electroanatomical mapping with a crista catheter is useful and time-saving in cases of RA tachycardias that have multiple origins or multiple reentrant circuits.

Recently, new 3D mapping technologies were developed, including CartoUnivu, CartoSound, and Paso. CartoUnivu is a fluoroscopy integrated 3D mapping system and contributed to a reduction in radiation exposure without compromising patient's safety [16]. CartoSound is the integration system of 3D electroanatomic mapping and intracardiac echocardiography. More accurate anatomic model and geometry can be obtained by CartoSound [17]. Paso module can deliver automated ECG-template matching during ventricular tachycardia mapping. If the newly developed $3 \mathrm{D}$ mapping technologies are combined with a crista catheter mapping, quick highresolution 3D mapping can be performed.

In patients with unusual heart anatomy, the ECG criteria for differentiating CTI-dependent and atypical atrial flutter are often not correct. In patients with atrial flutter, it is important to record two electrical sequences in the anterior and posterior portions of the RA lateral wall with a multi-electrode catheter, such as a crista catheter. We can then have an important clue to 
differentiate between CTI-dependent and scar-related atrial flutter by comparing the sequences of the two atrial electrograms. When the two sequences are the same, the probability of CTI-dependent atrial flutter is high and tachycardia could be easily terminated by CTI block without 3D electroanatomical mapping. If we can find the turnaround point of the electrical signal at the inferior margin of the right atriotomy scar, by thorough mapping with a multi-electrode catheter (e.g., a crista catheter), in patients with scar-related atrial flutter, we can perform RFCA successfully even without $3 \mathrm{D}$ electroanatomical mapping.

Because three or four electrophysiological catheters have to pass through the RA or occupy space within it, it is difficult to move the crista catheter or the lasso catheter freely in the RA. If we place a His-RV-type catheter and a decapolar catheter into the coronary sinus via the femoral vein, we can make enough space in the RA to move a crista catheter freely (Fig. 1a). Although in the early period we performed an electrophysiological study with various catheter positions, we found out that this catheter position was convenient for mapping the RA.

In the present study, the fluoroscopy time and procedure time in the crista group were not significantly shorter than those in the lasso group, although the 3D mapping speed in the crista group was significantly faster than that in the lasso group. This could have been because there were more patients with complex congenital heart disease in the crista group than in the lasso group.

\subsection{Study limitations}

The number of patients in the present study was small. Operator-dependency could not be totally excluded because electrophysiological studies were not performed by a single operator. In 7 of 20 patients with atrial flutter, intracardiac electrograms in the anterior and posterior portions of the RA lateral wall were not recorded. The crista catheter mapping technique needs to be tested by a small number of operators in a large number of patients.

\section{Conclusions}

A crista catheter is useful for high-density 3D electroanatomical mapping of complex RA tachycardia or flutter. Comparison of two electrical sequences from the anterior and posterior portions of the RA lateral wall can be helpful in differentiating between CTI-dependent and atypical atrial flutter.

Acknowledgements The authors thank Jungkee Lee from St. Jude Medical Korea Inc. and Jiwoon Jung from Biosense Webster Inc. for their 3D electroanatomical mapping with EnSite NavX and CARTO, respectively. The authors thank Mi Kyung Song MS, and Bo Kyung Ma, MS from Biostatistics Collaboration Unit, Yonsei University College of Medicine, for their statistical analysis.

Funding This work was not supported by any funding.

Conflict of interest The authors declare that they have no competing interests.

Ethical approval All procedures performed in the present study were in accordance with the ethical standards of the institutional research committee and with the 1964 Helsinki declaration and its later amendments. Formal informed consents were exempted by the board because this was a retrospective study.

Author contributions Jae-Sun Uhm was responsible for the concept/ design, data analysis/interpretation, drifting article, approval of the article, statistics, and data collection. Nam Kyun Kim was responsible for the concept/design, data analysis/interpretation, critical revision of the article, approval of the article, statistics, and data collection. Hancheol Lee was responsible for data analysis/interpretation, critical revision of the article, approval of the article, and data collection. Tae-Hoon Kim was responsible for data analysis/interpretation, critical revision of the article, approval of the article, and data collection. Boyoung Joung was responsible for data analysis/interpretation, critical revision of the article, approval of the article, and data collection. Hui-Nam Pak was responsible for data analysis/interpretation, critical revision of the article, approval of the article, and data collection. Moon-Hyoung Lee was responsible for the concept/ design, data analysis/interpretation, critical revision of the article, approval of the article, and data collection.

Open Access This article is distributed under the terms of the Creative Commons Attribution 4.0 International License (http://creativecommons.org/licenses/by/4.0/), which permits unrestricted use, distribution, and reproduction in any medium, provided you give appropriate credit to the original author(s) and the source, provide a link to the Creative Commons license, and indicate if changes were made.

\section{References}

1. Kistler, P. M., Roberts-Thomson, K. C., Haqqani, H. M., Fynn, S. P., Singarayar, S., Vohra, J. K., et al. (2006). P-wave morphology in focal atrial tachycardia: development of an algorithm to predict the anatomic site of origin. Journal of the American College of Cardiology, 48, 1010-1017.

2. Uhm, J.-S., Shim, J., Wi, J., Mun, H.-S., Pak, H.-N., Lee, M.,.-H., et al. (2014). An electrocardiography algorithm combined with clinical features could localize the origins of focal atrial tachycardias in adjacent structures. Europace, 16, 1061-1068.

3. Sherwin, E. D., Triedman, J. K., \& Walsh, E. P. (2013). Update on interventional electrophysiology in congenital heart disease: evolving solutions for complex hearts. Circulation: Arrhythmia and Electrophysiology, 6, 1032-1040.

4. Roberts-Thomson, K. C., Kistler, P. M., \& Kalman, J. M. (2006). Focal atrial tachycardia I: clinical features, diagnosis, mechanisms, and anatomic location. Pacing and Clinical Electrophysiology, 29, 643-652.

5. Wellens, H. J. (1994). Atrial tachycardia. How important is the mechanism? Circulation, 90, 1576-1577.

6. Saul, J. P., Walsh, E. P., \& Triedman, J. K. (1995). Mechanisms and therapy of complex arrhythmias in pediatric patients. Journal of Cardiovascular Electrophysiology, 6, 1129-1148. 
7. Kottkamp, H., Hindricks, G., Breithardt, G., \& Borggrefe, M. (1997). Three-dimensional electromagnetic catheter technology: electroanatomical mapping of the right atrium and ablation of ectopic atrial tachycardia. Journal Cardiovascular Electrophysiology, 8, 1332-1337.

8. Lukac, P., Pedersen, A. K., Mortensen, P. T., Jensen, H. K., Hjortdal, V., \& Hansen, P. S. (2005). Ablation of atrial tachycardia after surgery for congenital and acquired heart disease using an electroanatomic mapping system: which circuits to expect in which substrate? Heart Rhythm, 2, 64-72.

9. Shah, D., Jais, P., Takahashi, A., Hocini, M., Peng, J. T., Clementy, J., et al. (2000). Dual-loop intra-atrial reentry in humans. Circulation, 101, 631-639.

10. Uhm, J.-S., Mun, H.-S., Wi, J., Shim, J., Hwang, H. J., Sung, J.-H., et al. (2012). Importance of tachycardia cycle length for differentiating typical atrial flutter from scar-related in adult congenital heart disease. Pacing and Clinical Electrophysiology, 35, 1338-1347.

11. Chan, D. P., Van Hare, G. F., Mackall, J. A., Carlson, M. D., \& Waldo, A. L. (2000). Importance of atrial flutter isthmus in postoperative intraatrial reentrant tachycardia. Circulation, 102, 1283-1289.

12. Jones, D. G., McCready, J. W., Kaba, R. A., Ahsan, S. Y., Lyne, J. C., Wang, J., et al. (2011). A multi-purpose spiral high-density mapping catheter: initial clinical experience in complex atrial arrhythmias. Journal of Interventional Cardiac Electrophysiology, 31, 225-235.

13. Patel, A. M., d'Avila, A., Neuzil, P., Kim, S. J., Mela, T., Singh, J. P., et al. (2008). Atrial tachycardia after ablation of persistent atrial fibrillation: identification of the critical isthmus with a combination of multielectrode activation mapping and targeted entrainment mapping. Circulation. Arrhythmia and Electrophysiology, 1, 1422.

14. Reddy, V. Y., Morales, G., Ahmed, H., Neuzil, P., Dukkipati, S., Kim, S., et al. (2010). Catheter ablation of atrial fibrillation without the use of fluoroscopy. Heart Rhythm, 7, 1644-1653.

15. Anter, E., Tschabrunn, C. M., \& Josephson, M. E. (2015). Highresolution mapping of scar-related atrial arrhythmias using smaller electrodes with closer interelectorode spacing. Circulation. Arrhythmia and Electrophysiology, 8, 537-545.

16. Christoph, M., Wunderlich, C., Moebius, S., Forkmann, M., Sitzy, J., Salmas, J., et al. (2015). Fluoroscopy integrated 3D mapping significantly reduces radiation exposure during ablation for a wide spectrum of cardiac arrhythmias. Europace, 17, 928-937.

17. Kean, A. C., Gelehrter, S. K., Shetty, I., Dick, M., II, \& Bradley, D. J. (2010). Experience with CartoSound for arrhythmia ablation in pediatric and congenital heart disease patients. Journal of Interventional Cardiac Electrophysiology, 29, 139-145. 